\title{
An Admission Control Strategy for Differentiated Services in IEEE 802.11
}

\author{
Yu-Liang Kuo*, Chi-Hung Lu*, Eric Hsiao-Kuang $\mathrm{Wu}^{\dagger}$, and Gen-Huey Chen* \\ * Dept. of Computer Science and Information Engineering, National Taiwan University, Taipei, Taiwan, R.O.C. \\ ${ }^{\dagger}$ Dept. of Computer Science and Information Engineering, National Central University, Chung-Li, Taiwan, R.O.C.
}

\begin{abstract}
With the provisioning of high-speed wireless LAN (WLAN) environments, traffic classes (e.g., VoIP or videoconference) with different $\mathrm{QoS}$ requirements will be introduced in future WLANs. The IEEE 802.11e draft is currently standardizing a distributed access approach, called the enhanced distributed coordination function (EDCF), to support service differentiation in the MAC layer. However, since each mobile station transmits data packets egotistically in a distributed environment, the QoS requirement of each traffic class may not be guaranteed. In this paper, we develop an admission control strategy to guarantee the $Q o S$ requirement of each traffic class. In order to provide a criterion for admission decision, we introduce an analytical model for EDCF to evaluate the expected bandwidth and the expected packet delay of each traffic class. The admission control strategy uses the performance measures derived from the analytical model to decide if a new traffic stream is permitted into the system. We validate the accuracy of the analytical model by using the $n s-2$ simulator. Some performance evaluations are also demonstrated to illustrate the effect of the proposed admission control strategy.
\end{abstract}

\section{INTRODUCTION}

Recent developments in the IEEE 802.11 standardizations [2] have been successful to offer high-speed data services. Hence, traffic classes (e.g., VoIP or video-conference) with different QoS requirements will be provided in future WLANs. Since these traffic classes require distinct specific features, such as delay-sensitivity or required bandwidth, it is desired to provide a service differentiation mechanism in the IEEE 802.11 standard. Providing service differentiation in a wireless environment requires the MAC protocol to support some degree of separation between different traffic classes. Recently, the IEEE 802.11e draft is specifying a distributed access approach, called EDCF, to support service differentiation in the MAC layer [4]. It ensures that the packets sent by each mobile station can be differentiated by assigning different access parameters.

However, supporting service differentiation in the MAC protocol does not guarantee that the QoS requirement of each traffic class will be fully satisfied. Since each mobile station may transmit packets egotistically in a distributed environment, radio performance might be led to an unacceptable level. An admission control strategy might alleviate the effect of egotistic transmissions. It could ensure that the acceptance of a new traffic stream will not cause the QoS of any ongoing sessions below an unacceptable level.

In this paper, we develop an admission control strategy to guarantee the QoS requirement of each traffic class. The service differentiation mechanism is based on EDCF and the environment we consider is a single cell coordinated by an access point (AP). In the proposed admission control strategy, mobile stations make use of some MAC management messages specified in the IEEE 802.11e draft to transmit load conditions to the corresponding AP. The AP executes the admission control algorithm to estimate the performance of resource usage and decides if a new traffic stream is permitted into the system. In order to provide a criterion for admission decision, we introduce an analytical model to evaluate the performance of resource usage in EDCF. This model can evaluate the expected bandwidth and the expected packet delay for each traffic class. We validate the accuracy of the model and evaluate the performance by using the $n s-2$ simulator [1]. Since the model is simple in calculating the desired results, the admission decision can be made in real time. The performance evaluations for EDCF have appeared in the recent literatures [8][9][10], but the results are via simulations, and no theoretical analysis is given. The model we derived also provides a theoretical analysis for EDCF.

The paper is organized as follows. Section II presents the distributed coordination function (DCF) in IEEE 802.11 and the EDCF that is currently specified in the IEEE 802.11e draft. Section III presents the analytical model for the service differentiation mechanism. The validation of our analytical model is also presented in Section III. We present the proposed admission control strategy and simulation results in Section IV. The conclusions are drawn in Section V.

\section{DCF AND EDCF}

\section{A. Distributed Coordination Function}

The fundamental access method in IEEE 802.11 is DCF, which is based on carrier sense multiple access with collision avoidance (CSMA/CA) protocol. A mobile station that intends to transmit a packet waits until the channel is sensed idle for a time period equal to the DCF interframe space (DIFS). If the channel is sensed idle for a duration of DIFS, then it can immediately transmit a packet. Otherwise, the mobile station will generate a backoff time counter. A discrete time counter is used and the time following an idle DIFS is slotted. A mobile station is allowed to transmit only at the beginning of a time slot.

When a mobile station senses the channel busy during the duration of DIFS, the backoff time counter is randomly selected from the range $(0, \mathrm{CW})$, where $\mathrm{CW}$ is called the contention window. At the first transmitting attempt, $\mathrm{CW}$ is 
TABLE I

The Four Default ACs SPeCified IN THE IEEE 802.11E

\begin{tabular}{ccccc}
\hline $\mathrm{AC}$ & AIFSD (AIFS) & $\mathrm{CW}_{\min }$ & $\mathrm{CW}_{\max }(m)$ & Designation \\
\hline $\mathrm{AC}_{0}$ & $34 \mu \mathrm{s} \mathrm{(2)}$ & 15 & $1023(6)$ & Best Effort \\
$\mathrm{AC}_{1}$ & $25 \mu \mathrm{s}(1)$ & 15 & $1023(6)$ & Video Probe \\
$\mathrm{AC}_{2}$ & $25 \mu \mathrm{s}(1)$ & 7 & $15(1)$ & Video \\
$\mathrm{AC}_{3}$ & $25 \mu \mathrm{s}(1)$ & 3 & $7(1)$ & Voice \\
\hline
\end{tabular}

assigned the value $\mathrm{CW}_{\min }$, which is called the minimum contention window. In the consecutive unsuccessful transmissions (due to collisions), the value of $\mathrm{CW}$ is increasing up to the maximum value $\mathrm{CW}_{\max }=2^{m} \mathrm{CW}_{\min }$, where $m$ is called the maximum backoff stage.

The backoff time counter is decreased as the channel is sensed idle and suspended as the channel is sensed busy. After the suspension, the counter is reactivated as the channel is again sensed idle for a duration of DIFS. The mobile station will transmit a packet when the counter reaches zero.

\section{B. Enhanced Distributed Coordinated Function}

The goal of EDCF is to provide a distributed access mechanism to support service differentiation. EDCF introduces the concept of access categories (ACs), which are variants of the DCF access mechanism. The IEEE 802.11e draft currently specifies four default ACs. The four default ACs are listed in Table I in which the IEEE 802.11a physical layer [3] is adopted. Different ACs use different values of arbitration interframe space duration (AIFSD), $\mathrm{CW}_{\min }$, and $\mathrm{CW}_{\max }$. Traffic classes with smaller values of $\mathrm{CW}_{\min }$ and $\mathrm{CW}_{\max }$ yield higher priorities. Furthermore, different interframe spaces can be used by different traffic classes. DCF Interframe space (DIFS) is substituted for the AIFSD. AIFSD is at least a duration of short interframe space (SIFS) plus a slot time and can be enlarged individually by different traffic classes. Let the length of a slot time be denoted by $\delta$. AIFSD can be computed as the following:

$$
\text { AIFSD }=\text { SIFS }+ \text { AIFS } \times \text { aSlotTime, }
$$

where AIFS is a positive integer which is equal to or greater than 1. Hence, AIFSD is determined by AIFS and traffic classes with smaller values of AIFS yield higher priorities. These three parameters are gathered and called a QoS parameter set.

In EDCF, data packets are delivered through multiple backoff instances within one mobile station. A single mobile station may implement up to 4 transmission queues and each transmission queue uses a specific AC for contending the channel access, as illustrated in Fig. 1. Each queue within the mobile station contends for the channel access and independently starts its backoff procedure depending on its associated AC. If the backoff time counters of two or more parallel queues within a single mobile station reach zero at the same time, a internal scheduler will resolve the internal collision. The

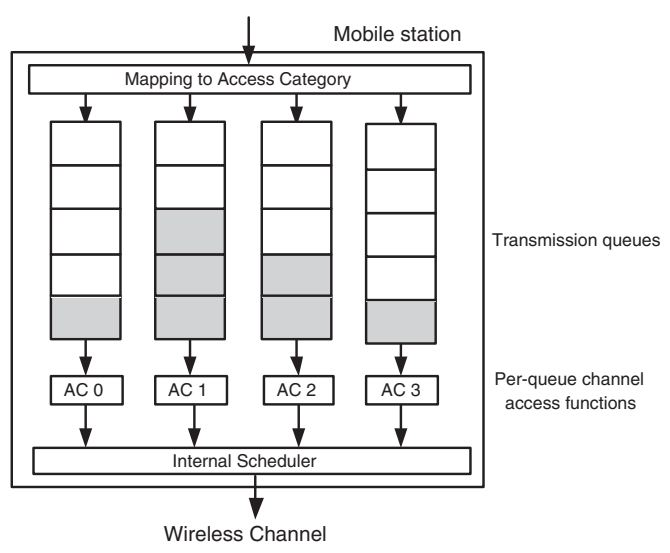

Fig. 1. A single mobile station can implement up to 4 transmission queues. Each queue is mapping to a particular access category.

scheduler grants the channel access to the queue in terms of its particular scheduling algorithm.

\section{Analytical Model And Model Validation}

The environment we consider is a single wireless cell coordinated by an AP. In a single cell environment, each mobile station which intends to transmit a packet needs to forward its packet to the corresponding AP. The transmitted packets should be forwarded to the AP even if the packets are destined to the mobile station in the same cell. We assume all mobile stations and the corresponding AP can communicate with each other without obstacle and there is no hidden terminal problem. The access mechanism we consider is a fourway handshaking protocol by using the RTS/CTS/DATA/ACK dialogue.

\section{A. Analytical Model}

Without loss of generality, we assume there are $K$ traffic classes with distinct QoS requirements. Specifically, there are $\mathbf{n}=\left(n_{0}, n_{1}, \ldots, n_{K-1}\right)$ mobile stations, where $n_{k}(0 \leq$ $k \leq K-1)$ is the number of mobile stations which generate traffic class $k$ packets. For convenience, let the traffic class $k$ packet be denoted by class- $k$ packet and the class- $k$ station is referred to as the mobile station that generates class- $k$ packets to transmit. Traffic class $k$ stations use $\mathrm{AC}_{k}$ to access the wireless channel. We assume that each class- $k$ packet has constant length (number of bits) $L_{k}$ and requires $\frac{L_{k}}{M}$ seconds for data transmission time, where $M$ is the average channel bit rate. We also assume that each mobile station always has a packet ready to transmit. In other words, we consider the saturation condition [6]. The maximum propagation delay for all packets is assumed to be a constant length of $\tau$ seconds.

At each transmission attempt, we assume that each class- $k$ packet has common probability $p_{k}$ of involving in collision when it is being transmitted, where $p_{k}$ is independent of retransmission history. The assumption of $p_{k}$ is originated from Bianchi's model [6] but we extend the assumption to allow $K$ traffic classes. Suppose that a class- $k$ station that has involved in $l$ times of collision will select the backoff time 
counter $B_{k_{l}}(l \geq 0)$ before (re)transmission. Let $J_{k}$ denote the number of collisions that a class- $k$ station has involved. As a class- $k$ station intends to transmit a packet, the expected backoff time counter $E\left[B_{k}\right]$ can be computed by conditioning on $J_{k}$

$$
E\left[B_{k}\right]=\sum_{l=0}^{\infty} E\left[B_{k_{l}}\right] P\left\{J_{k}=l\right\} .
$$

The distribution of $J_{k}$ is

$$
P\left\{J_{k}=l\right\}= \begin{cases}p_{k}^{l} & \text { if } l \geq 1, \\ 1-\sum_{i=1}^{\infty} p_{k}^{i} & \text { if } l=0 .\end{cases}
$$

Let $W_{k}, \mathrm{AIFS}_{k}$, and $m_{k}$ be the minimum contention window, AIFS, and maximum backoff stage for each class$k$ station. Since $B_{k_{l}}$ is selected from the current contention window (the current contention window is obtained according to the binary exponential backoff procedure) in a uniformed way, its probability mass function is

$$
\begin{gathered}
P\left\{B_{k_{l}}=i\right\}=\frac{1}{2^{\beta_{k}(l)}\left(\mathrm{W}_{k}+1\right)+\mathrm{AIFS}_{k}}, \\
i=0,1, \cdots, 2^{\beta_{k}(l)}\left(\mathrm{W}_{k}+1\right)+\mathrm{AIFS}_{k}-1,
\end{gathered}
$$

where $\beta(\cdot)$ is defined as the following:

$$
\beta_{k}(i)= \begin{cases}i & \text { if } 0 \leq i \leq m_{k}-1, \\ m_{k} & \text { if } i \geq m_{k} .\end{cases}
$$

The expectation of $B_{k_{l}}$ can be easily derived from (3)

$$
E\left[B_{k_{l}}\right]=\frac{2^{\beta_{k}(l)}\left(\mathrm{W}_{k}+1\right)+\mathrm{AIFS}_{k}-1}{2} .
$$

According to (2) and (4), (1) can be expressed as

$$
\begin{aligned}
E\left[B_{k}\right]= & \left(1-\sum_{i=1}^{\infty} p_{k}^{i}\right) \frac{W_{k}+\mathrm{AIFS}_{k}}{2}+ \\
& \sum_{i=1}^{\infty} p_{k}^{i} \frac{2^{\beta_{k}(i)}\left(\mathrm{W}_{k}+1\right)+\mathrm{AIFS}_{k}-1}{2} .
\end{aligned}
$$

At a given time slot, the probability that a class- $k$ station will transmit is [7]

$$
q_{k}=\frac{1}{E\left[B_{k}\right]+1} .
$$

According to (6), $p_{k}$ can be computed as

$$
p_{k}=\left(1-\left(1-q_{k}\right)^{n_{k}-1} \prod_{j=0, j \neq k}^{K-1}\left(1-q_{j}\right)^{n_{j}}\right) .
$$

By observing the transmission behavior in the wireless channel, a pattern of periodical cycles can be found. Each cycle, named as transmission cycle, consists of some idle periods, some unsuccessful periods (due to collision or error), and a successful period, as depicted in Fig. 2. When each cycle ends in a successful period, a consecutive cycle will restart with respect to the ordered sequences (idle periods, unsuccessful periods, a successful period). Let $E[I], E[C]$, and $E[S]$ be the sum of the expected lengths of all idle

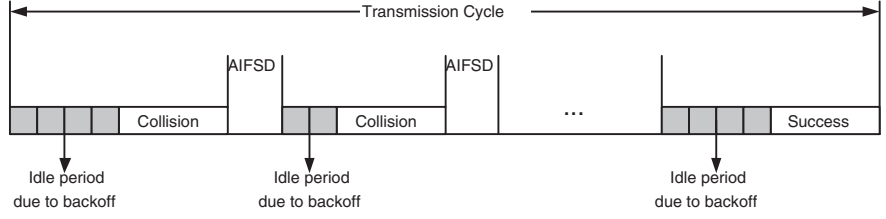

Fig. 2. A transmission cycle.

periods, all unsuccessful periods, and the expected length of a successful period, respectively. In addition, let $E\left[L_{k}\right]$ be the expected proportional number of bits a class- $k$ packet will be transmitted in a successful period. As a result, using renewal theory [11], the expected bandwidth for class- $k$ stations is given as

$$
\rho_{k}=\frac{E\left[L_{k}\right]}{E[I]+E[C]+E[S]} .
$$

The probability that a class- $k$ packet will be transmitted in the successful period of a transmission cycle is

$\gamma_{k}=\mathrm{P}\{$ Transmitting station $=1$ and is class- $k$ station $\mid$

$$
\text { Transmitting station } \geq 1 \text { \}. }
$$

Hence (9) can be computed as

$$
\gamma_{k}=\frac{n_{k} q_{k}\left(1-q_{k}\right)^{n_{k}-1} \prod_{i=0, i \neq k}^{K-1}\left(1-q_{i}\right)^{n_{i}}}{1-\prod_{i=0}^{K-1}\left(1-q_{i}\right)^{n_{i}}} .
$$

Since only one particular class- $k$ packet can be transmitted in the successful period of a transmission cycle, we compute $E\left[L_{k}\right]$ according to the normalized probability $\gamma_{k}$. Thus

$$
E\left[L_{k}\right]=\frac{\gamma_{k}}{\gamma_{0}+\gamma_{1}+\cdots+\gamma_{K-1}} L_{k}
$$

Also, the expected proportional AIFS a class- $k$ packet required in the successful period of a transmission cycle is given as

$$
E\left[\mathrm{AIFS}_{k}\right]=\frac{\gamma_{k}}{\gamma_{0}+\gamma_{1}+\cdots+\gamma_{K-1}} \text { AIFS }_{k} .
$$

The expected number of bits transmitted in a successful period is computed as $E[L]=\sum_{k=0}^{K-1} E\left[L_{k}\right]$ and the expected AIFS required in a successful period is computed as $E[A]=$ $\sum_{k=0}^{K-1} E\left[\mathrm{AIFS}_{k}\right]$.

Let $T_{S I F S}, T_{A C K}, T_{R T S}, T_{C T S}$, and $H$ be the duration required for a SIFS, transmitting a ACK, transmitting a RTS, transmitting a CTS, and transmitting a PHY/MAC header, respectively. The expected length of a successful period in a transmission cycle is approximately expressed as

$E[S]=T_{R T S}+T_{C T S}+T_{A C K}+\frac{E[L]}{M}+4 \tau+4 T_{S I F S}+\delta E[A]+H$.

Given $\mathbf{n}=\left(n_{0}, n_{1}, \ldots, n_{K-1}\right)$, we let $N_{c}$ be the random variable representing the number of colliding periods needed in a transmission cycle. The distribution of $N_{c}$ is geometric with parameter $\left(1-p_{c}\right)$, where $p_{c}$ is the collision probability viewed by the channel and can be computed as

$P\{$ Transmitting station $\geq 2 \mid$ Transmitting station $\geq 1\}=$ $1-P\{$ Transmitting station $=0\}-P\{$ Transmitting station $=1\}$ $P\{$ Transmitting station $\geq 1\}$ 
From (14), $p_{c}$ can be expressed as

$$
p_{c}=\frac{1-\prod_{i=0}^{K-1}\left(1-q_{i}\right)^{n_{i}}-X}{1-\prod_{i=0}^{K-1}\left(1-q_{i}\right)^{n_{i}}},
$$

where $X=\sum_{i=0}^{K-1} n_{i} q_{i}\left(1-q_{i}\right)^{n_{i}-1} \prod_{j=0, j \neq i}^{K-1}\left(1-q_{j}\right)^{n_{j}}$. As a result, we have

$$
E\left[N_{c}\right]=\frac{p_{c}}{1-p_{c}} .
$$

The sum of the expected lengths of all unsuccessful periods in a transmission cycle is

$$
E[C]=E\left[N_{c}\right]\left(\tau+T_{S I F S}+\delta E[A]+T_{R T S}+H\right) .
$$

This follows since the length of an unsuccessful period is $\tau+$ $T_{S I F S}+\delta E[A]+T_{R T S}+H$ and the expected number of unsuccessful periods is $E\left[N_{c}\right]$.

The number of time slots required for an idle period in a transmission cycle can be viewed as a geometric random variable with parameter $\left(1-\prod_{i=1}^{K}\left(1-q_{i}\right)^{n_{i}}\right)$. Hence, the expected length of an idle period can be computed as

$$
\begin{array}{r}
\delta \sum_{j=0}^{\infty} j\left(1-\prod_{i=0}^{K-1}\left(1-q_{i}\right)^{n_{i}}\right)\left(\prod_{i=0}^{K-1}\left(1-q_{i}\right)^{n_{i}}\right)^{j} \\
=\left(\frac{\delta \prod_{i=0}^{K-1}\left(1-q_{i}\right)^{n_{i}}}{1-\prod_{i=0}^{K-1}\left(1-q_{i}\right)^{n_{i}}}\right) .
\end{array}
$$

The lengths of all idle periods are assumed to be identical and independent distribution and there are $E\left[N_{c}\right]+1$ idle periods in a transmission cycle. The sum of the expected lengths of all idle periods in a transmission cycle is

$$
E[I]=\left(E\left[N_{c}\right]+1\right)\left(\frac{\delta \prod_{i=0}^{K-1}\left(1-q_{i}\right)^{n_{i}}}{1-\prod_{i=0}^{K-1}\left(1-q_{i}\right)^{n_{i}}}\right) .
$$

In this paper, the expected packet delay for a class- $k$ packet is defined as the time between the arrival of a class$k$ packet and its successful transmission to the receiver. The expected packet delay is composed of the access delay and the transmission delay. The access delay is the time interval from the packet arriving at the station to the packet being ready for acquiring the channel access. The transmission delay is the time interval from the packet being ready for acquiring the channel access to the packet being successfully received by the receiver. We assume that all stations have infinite queue length. Hence we can compute the expect packet delay for a class- $k$ packet by using G/G/1 model [5]. The access and transmission delays of a class- $k$ packet can be viewed as waiting time in queue and service time respectively in a queueing system. According to G/G/1 model, the average waiting time in queue (access delay) for a class- $k$ packet satisfies

$$
E\left[Q_{k}\right] \leq \frac{\lambda_{k}\left(\sigma_{a_{k}}^{2}+\sigma_{b_{k}}^{2}\right)}{2\left(1-\lambda_{k} E\left[T_{k}\right]\right)}
$$

where

- $\lambda_{k}$ - average packet inter-arrival time for class- $k$ packets

- $E\left[T_{k}\right]$ - average transmission delay for a class- $k$ packet,
TABLE II

THE VALUES OF PARAMETERS USED IN MODEL VALIDATION

\begin{tabular}{cc}
\hline FTP packet payload size $\left(\mathrm{AC}_{0}\right)$ & $1500 \mathrm{bytes}$ \\
Video packet payload size $\left(\mathrm{AC}_{2}\right)$ & $1464 \mathrm{bytes}$ \\
Voice packet payload size $\left(\mathrm{AC}_{3}\right)$ & $96 \mathrm{bytes}$ \\
average packet inter-arrival time $\lambda_{0}$ for FTP & $0.012 \mathrm{pkt} / \mathrm{sec}$ \\
average packet inter-arrival time $\lambda_{2}$ for video & $0.01 \mathrm{pkt} / \mathrm{sec}$ \\
average packet inter-arrival time $\lambda_{3}$ for voice & $0.02 \mathrm{pkt} / \mathrm{sec}$ \\
PHY header & $6 \mathrm{bytes}$ \\
MAC header & $34 \mathrm{bytes}$ \\
RTS & $20 \mathrm{bytes}$ \\
CTS & $14 \mathrm{bytes}$ \\
ACK & $14 \mathrm{bytes}$ \\
Propagation delay & $1 \mu \mathrm{s}$ \\
Average channel bit rate & $1 \mathrm{Mbps}$ \\
SIFS & $16 \mu \mathrm{s}$ \\
Slot time & $9 \mu \mathrm{s}$ \\
channel bit error rate & $10^{-5}$ \\
\hline
\end{tabular}

- $\sigma_{a_{k}}^{2}$ - variance of packet inter-arrival times for class- $k$ packets,

- $\sigma_{b_{k}}^{2}$ - variance of transmission delays for class- $k$ packets.

Assuming that the channel bit error rate in the wireless medium is $p_{b}$. The successful transmission probability without error-prone for a class- $k$ packet is (1 $\left.p_{b}\right)^{L_{k}}$. $E\left[T_{k}\right]$ can be verbally represented as (The time required for retransmission $) \times$ (Average number of retransmission)+(Successful data transmission time). As a result, we have

$$
\begin{aligned}
E\left[T_{k}\right] & =a_{k} E\left[J_{k}\right]+\left(1-p_{b}\right)^{L_{k}} b_{k}+\left(1-\left(1-p_{b}\right)^{L_{k}}\right) E\left[T_{k}\right] \\
& =\frac{a_{k}}{\left(1-p_{b}\right)^{L_{k}}} E\left[J_{k}\right]+b_{k} .
\end{aligned}
$$

where $a_{k}=\delta E\left[B_{k}\right]+T_{R T S}+\tau+2 T_{S I F S}+\delta \mathrm{AIFS}_{k}$ and $b_{k}=\delta E\left[B_{k}\right]+H+\delta \operatorname{AIFS}_{k}+T_{R T S}+T_{C T S}+\frac{L_{k}}{M}+T_{A C K}+$ $4 T_{S I F S}+4 \tau$. According to (20) and (21), the expected packet delay for a class- $k$ packet can be expressed as

$$
E\left[D_{k}\right]=E\left[Q_{k}\right]+E\left[T_{k}\right] .
$$

\section{B. Model Validation}

We validate the analytical model by using the $n s-2$ simulator. The values of parameters used in the analytical and simulative models are summarized in Table II. In this validation, each class- $k$ packet has constant packet payload size, as shown in Table II. We have three common applications (FTP, video, and voice applications) and each one is associated with default AC specified in the IEEE 802.11e draft. FTP, video, and voice applications are associated with $\mathrm{AC}_{0}, \mathrm{AC}_{2}, \mathrm{AC}_{3}$ respectively for acquiring channel access. The values of parameters are assigned depending on the IEEE 802.11a specification [3]. For simulation efficiency and simplicity, the average channel bit rate is assumed to be $1 \mathrm{Mbps}$.

In the model validation, we have three mobile stations. These three mobile stations perform FTP, video, and voice applications, respectively. Fig. 3(a) shows the bandwidth results 


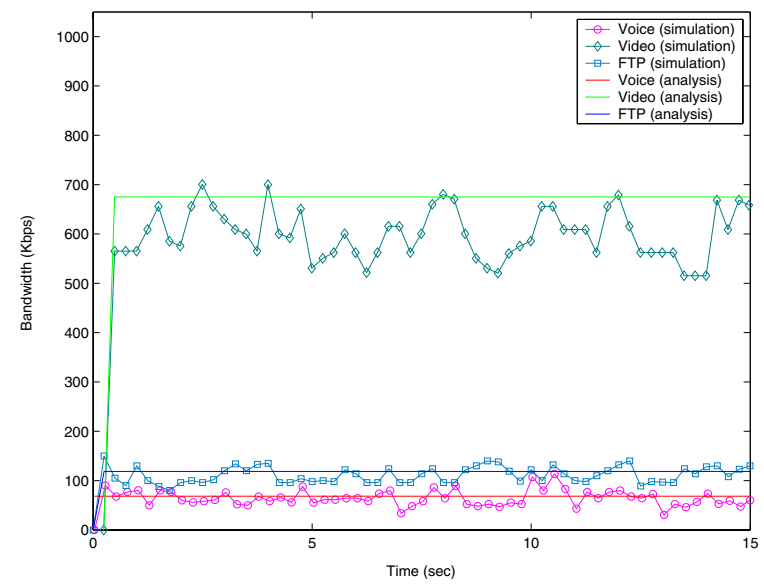

(a) Expected bandwidth.

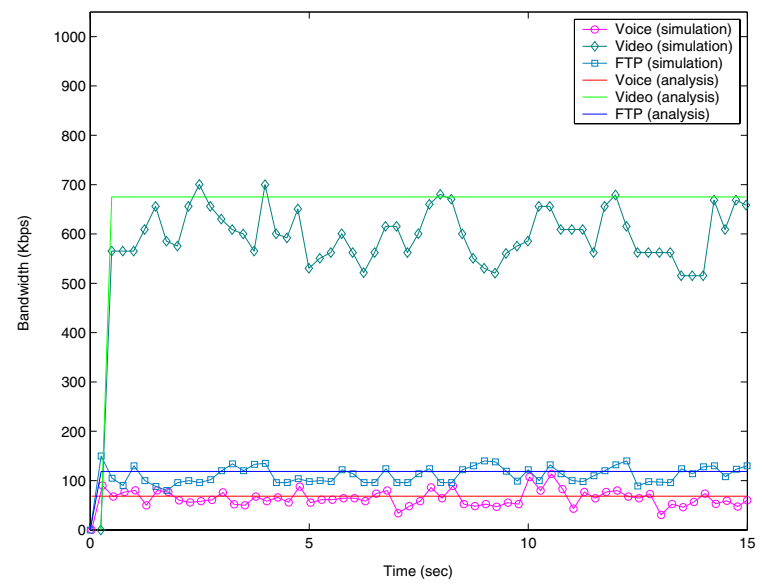

(b) Expected packet delay.

Fig. 3. Analytical model vs. simulative model (one FTP, one video, and one voice applications).

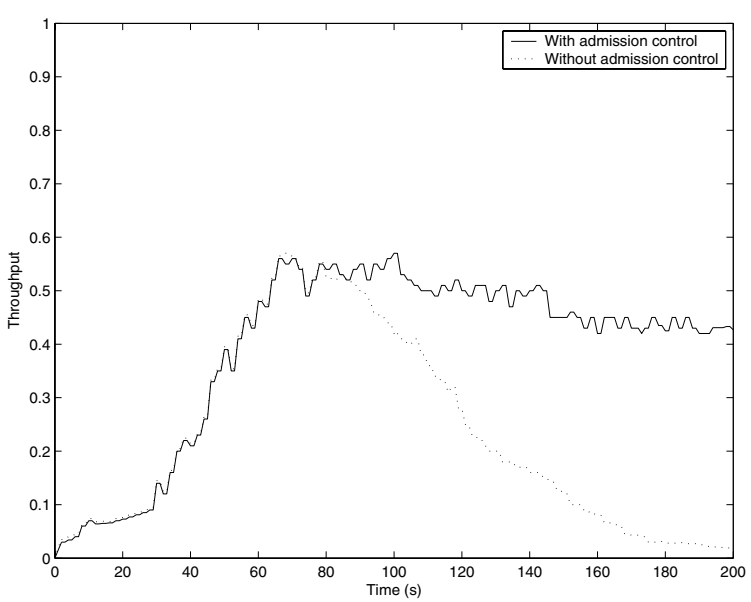

(a) Throughput for traffic class 2 .

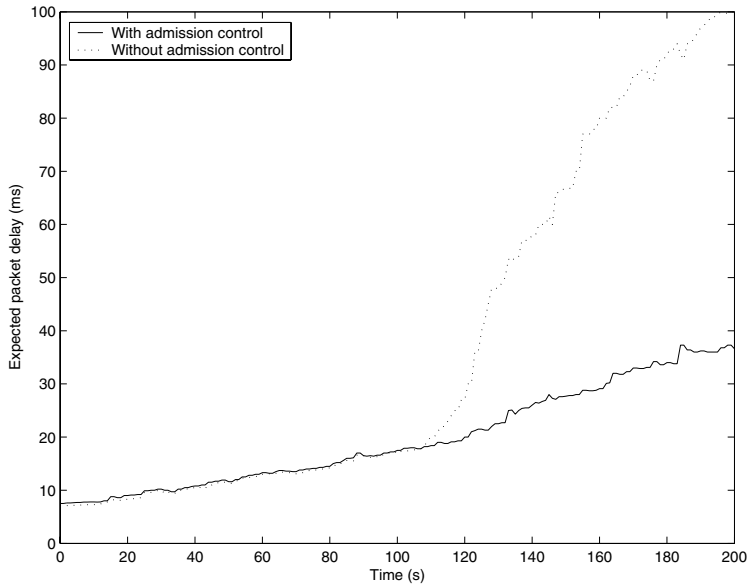

(b) Expected packet delay for traffic class 2 .

Fig. 4. Performance evaluations for the proposed admission control strategy.

of the simulative model, in which diamond line represents simulative bandwidth of video, square line represents simulative bandwidth of FTP, and circle line represents simulative bandwidth of voice, are close to the expected bandwidth results of the analytical model (solid lines).

The packet inter-arrival times of FTP, video, and voice applications follow the exponential distribution, general distribution, and deterministic distribution, respectively. The average packet inter-arrival times for these three applications are listed in Table II. Fig. 3(b) shows the delay results of the simulative and analytical models. The validation shows that the expected packet delays of the analytical model (solid lines) are also close to the one of simulative model.

\section{The Proposed AdMission CONTROL STRATEGy AND SIMULATION RESULTS}

\section{A. The Proposed Admission Control Strategy}

We assume that the QoS requirement for traffic class $k$ consists of the following items:
- minimum data rate $\left(\mathrm{MinDR}_{k}\right)$,

- maximum data rate $\left(\mathrm{MaxDR}_{k}\right)$,

- maximum tolerable packet delay $\left(\right.$ Delay $\left._{k}\right)$, and

- maximum tolerate packet loss rate $\left(\mathrm{LR}_{k}\right)$.

The average data rate can be computed as $\operatorname{AvgDR}_{k}=$ $\frac{\operatorname{MaxDR}_{k}+\mathrm{MinDR}_{k}}{2}$. The objective of admission control is to guarantee the required bandwidths of all traffic class are satisfied. In addition, the expected packet delay for each traffic class $k$ will not exceed Delay $_{k}$.

The proposed admission control algorithm is implemented in APs. The AP should collect information of load conditions from mobile stations to estimate the radio performance. We make use of some MAC management messages specified in the IEEE 802.11e draft to transmit the required information. Each mobile station first transmits a ADDTS request message to the AP before transmitting a traffic stream (TS). A TS is a set of certain traffic class packets. The ADDTS request message contains the traffic class information of the TS and the MAC address of this mobile station. 
Upon receipt of the request message, the AP estimates the radio performance by using the analytical model described in III-A. The proposed algorithm use the performance measures derived by the analytical model to see if the QoS requirements for all mobile stations can be guaranteed after the TS is permitted into the system. As a $k$-class station issues a ADDTS request message, the admission control algorithm will be triggered, as shown in Algorithm 1. If the QoS requirements are guaranteed for all mobile stations, then the AP responds a ADDTS response message with acceptance status to notify the mobile station of approving the TS into the system. Otherwise, the AP will respond a ADDTS response message with rejection status to notify the mobile station of rejecting the TS into the system. After the mobile station receives the ADDTS response message, it can transmit packets until there is no packet in the transmission queue of the mobile station. As the transmission queue of the mobile station gets empty, it will transmit a DELTS message to notify the AP of successful transmission of the TS. Upon receipt of the DELTS message, the AP responds a ACK to confirm the receipt. When there is another TS to be transmitted in the transmission queue, it should again issue a ADDTS request message for requesting admission. According to these messages (ADDTS request and DELTS message), the AP can obtain load conditions in the wireless medium and make precise estimation for the radio performance.

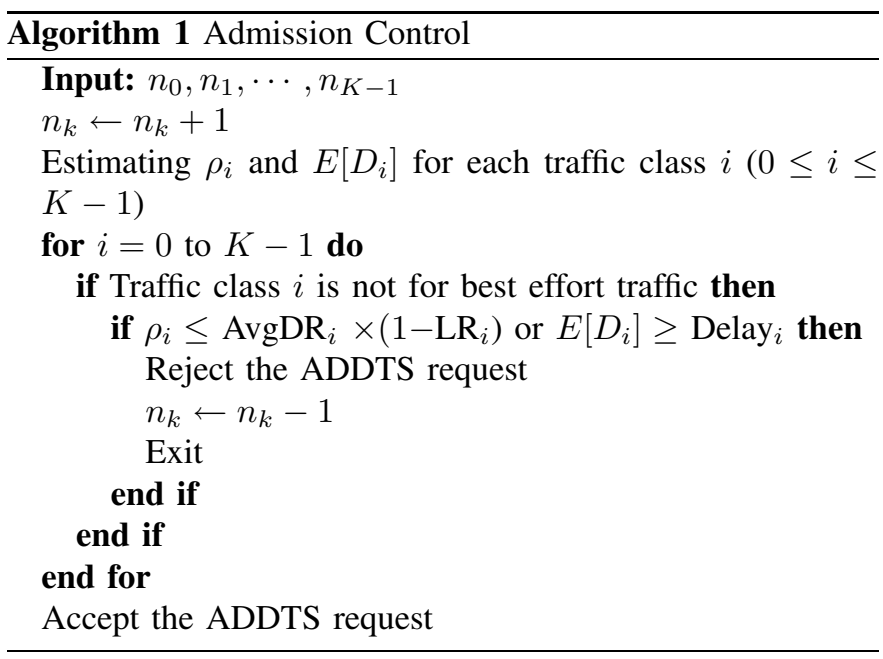

\section{B. Simulation Results}

We made an experiment to demonstrate the effect of our proposed admission control strategy. The values of parameters in the simulation environment are the same as model validation, which is listed in Table II. We consider the scenario that the number of the video flow is increasing, while the numbers of FTP and voice flows stay constant.

In this simulation, the traffic load is increasing by adding a new video flows periodically every 5 seconds. The solid line depicted in Fig. 4(a) represents the throughput for video applications with admission control, while the circle line represents the one without admission control. With the increment in the number of video flows, the admission control strategy could keep the throughput stable. Similarly, with applying the admission control strategy, the expected packet delay is stable, which is independent of load conditions, as depicted in Fig. 4(b).

\section{CONCLUSION}

The contribution of this paper is as follows. First, we introduced an analytical model to evaluate the expected bandwidth and the expected packet delay of each traffic class for the service differentiation mechanism based on EDCF. This model provides a criterion for admission decision as well as a theoretical analysis for EDCF. We validated this model by using the $n s-2$ simulator and the validation showed that the estimation of the analytical model is accurate. Second, the proposed admission control strategy satisfies the required bandwidth of each traffic class and limits the packet delay of each traffic class to a predefined level which is independent of load conditions. Therefore, the diverse QoS requirements in each traffic class can be fulfilled. In addition, the admission decision can be made in real time due to the simplicity of the analytical model. In the further research, we will investigate the effect of the hidden terminal problem and the dynamic tuning of ACs to optimize the system performance under various network conditions.

\section{ACKNOWLEDGMENT}

This research was supported by Ministry of Education, ROC, with contract No: 91 A-H-FA07-1-4 (Learning Technology) and the Communications Software Technology project of Institute for Information Industry and sponsored by MOEA ,R.O.C

\section{REFERENCES}

[1] "The Network Simulator - ns-2," http://www.isi.edu/nsnam/ns/.

[2] Wireless LAN Medium Access Control (MAC) and Physical Layer (PHY) Specification, IEEE 802.11 WG, Aug. 1999.

[3] Wireless LAN Medium Access Control (MAC) and Physical Layer (PHY) Specifications: High-speed Physical Layer in the $5 \mathrm{GHz}$ Band, IEEE Std 802.11a-1999, Sep. 1999.

[4] Wireless LAN Medium Access Control (MAC) and Physical Layer (PHY) Specification: Medium Access Control (MAC) Enhancements for Quality of Service $(Q o S)$, IEEE Std. 802.11e/D4.0 , Nov. 2002.

[5] D. Bertsekas and R. Gallager, Data Networks, (Englewood Cliffs, NJ: Prentice Hall, 2nd ed., 1992.)

[6] G. Bianchi, "Performance analysis of the IEEE 802.11 distributed coordination function," IEEE Journal of Selected Areas on Communication vol.18, no.3, pp. 535-547, March 2000.

[7] F. Cali, M. Conti and E. Gregori, "Dynamic Tuning of the IEEE 802.11 Protocol to Achieve a Theoretical Throughput Limit,' IEEE/ACM Trans. on Networking, vol. 8, pp. 785-799, Dec. 2000.

[8] P. Garg, R. Doshi, R. Greene, M. Baker, M. Malek, and X. Cheng, "Using IEEE 802.11e MAC for QoS over Wireless." to appear in Proc. IPCCC, Phoenix, Arizona, Apr. 2003.

[9] A. Lindgren, A. Almquist, O. Schelen, "Evaluation of Quality of Service Schemes for IEEE 802.11 Wireless LANs," in Proc. LCN, Tampa, Florida, Jul. 2001, pp. 348-351.

[10] S. Mangold, S. Choi, P. May, O. Klein, G. Hiertz, L. Stibor, "IEEE 802.11e Wireless LAN for Quality of Service," in Proc. European Wireless, vol. 1, Florence, Italy, Feb. 2002, pp. 32-39.

[11] S. M. Ross, Introduction to Probability Models, (Academic Press, 8nd ed., 2003.) 